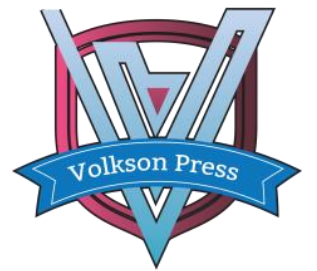

Contents List available at VOLKSON PRESS

Economics \& Management Innovations(EMI)

DOI : http://doi.org/10.26480/icemi.01.2017.367.369

\title{
Auto Active Airflow-Fan Cooler System Using Internet of Things Technology
}

\author{
Phumipat Niyomtham, Narongdech Keeratipranon and Ratthaslip Ranokphanuwat \\ * College of Innovative Technology and Engineering Dhurakij Pundit University Bangkok Thailand \\ *Email: udom.ran@dpu.ac.th
}

This is an open access article distributed under the Creative Commons Attribution License, which permits unrestricted use, distribution, and reproduction in any medium, provided the original work is properly cited.

\section{ARTICLE DETAILS}

\section{Article History:}

\section{Received 02 october 2017}

Accepted 06 october 2017

Available online 11 october 2017

\section{Keywords:}

Internet of Things, Amazon Web

Service, Cloud technology, Sensor, Gateway, Cooling system.

\section{ABSTRACT}

This paper is a study and development of a cooling system in automatic control to help solve common household problems, called an Auto Active Airflow-Fan Cooler System, especially in a city surrounded by concrete forests, which are very hot during the daytime. Moreover, heat transfers into a building, for example, a wall, floor or a window, and then kept inside the building. As a result, the building accumulates heat. In addition, the air conditioning results in slower working conditions and uses grater power consumption. The Auto Active AirflowFan Cooler System has a mechanism that allows for good ventilation, where windows are closed. The system will automatically run when the house temperature reaches a set point. The evening garden system will continue to work to provide effective ventilation, and will drip during the night, when the temperature in a building drops to a set level. If the Auto Active Airflow-Fan Cooler System is installed in conjunction with the ceiling insulation, it will help to improve the performance. The system developed using Internet of Things (IoT) and Cloud (AWS) technology. The IoT is designed and implemented using low cost Arduino + NRF240L1 technology for automatic temperature measurement, cooling the air in the building, and transmitting/receiving information to/from Cloud via the internet. Cloud (AWS) is used to operate automatically and deciding to run a ventilated fan works. It includes AWS IoT, DynamoDB and EC2 services. The system features are low cost. This technology automatically monitors manages the energy inside the building via web applications and mobile phones.

\section{Introduction}

Thailand is one of the world's top ten hotspots. Innovative home cooling, or "cool home", is a good solution. The overall climate trend is getting warmer every year. Good house design in today's climate is a process that can reduce the house temperature. At present, many construction material suppliers choose to innovate that can help keep the house cool in the cold, such as roofing materials, thermal insulation and aerated brick wall. Moreover, the design of the house in accordance with wind direction and sunlight becomes more important. However, there are limits to the design when changing lifestyles, especially of a houses located with

designs such that, they cannot open.

Nowadays, the innovation of ventilation and cooling of a house and the roof becomes too expensive. We have invented the new Active Air Flow System that keeps the home temperature cool. In this paper, we focus on experiments on simulators to study the process and work process through the Internet of Things (IoT) and Cloud (AWS) technology. Our contributions are as follows:

1. To design and develop an automatic cooling control system.

2. To design and develop a system for controlling and displaying fan on and off via web application or mobile application.

The rest of the paper is organized as follows. Section 2 presents the model and discusses the implementation details. Section 3 describes the results and discussions. Finally, Section 4 concludes and suggests future work.

\section{The Auto Active Airflow-Fan Cooler Model}

The ventilation system is designed to have an external air intake by means of a fan motor controlled by the temperature installed inside. There is a vent for exhaust air at the top, with a temperature sensor that determines the speed of the fan, both above and below. If the temperature is less than 32 degrees, the system will not work. When the temperature is more than
35 degrees, the system will control the fan to take the air out and drain it At a speed of $50 \mathrm{rpm}$, and when the temperature exceeds 45 degrees, the system speeds up to $200 \mathrm{rpm}$, with the fan having a separate control knob to separate the two fans. Working on both nodes sends the temperature and fan speed to the Node MCU to send up the AWS IoT.

In the case of AWS IoT data, there is a rule to control the value stored in the DynamoDB data base. The stored value is the temperature and fan speed with the timestamp specified. The Node MCU sends the value to the Cloud when the temperature changes. To save energy, the benefit of the following steps is as shown in Figure 1

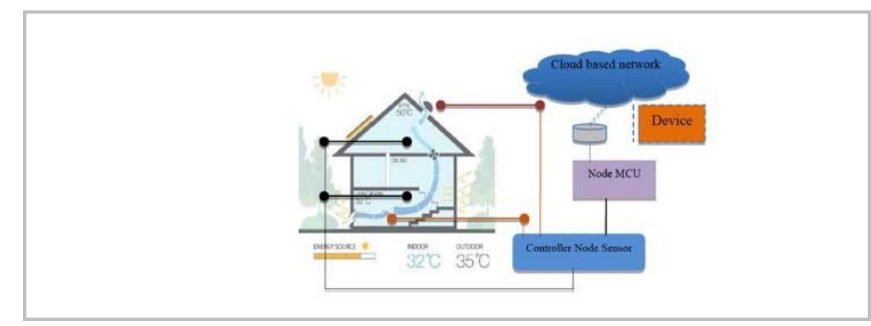

Figure 1. Active Airflow-Fan Cooler Model Overview

The model consists of three key components (according to the IoT concept [1][2]), where each component is assigned to any functional. The Arduino (Device) received sensor value from any sensor, after that send data to NodeMCU and NodeMCU (Gateway) send data to AWS Cloud, with MQTT protocol, which the data store in DynamoDB and executed by EC2 (Compute Cloud Service) on web browser with HTTP protocol as shown in Figure 2. 


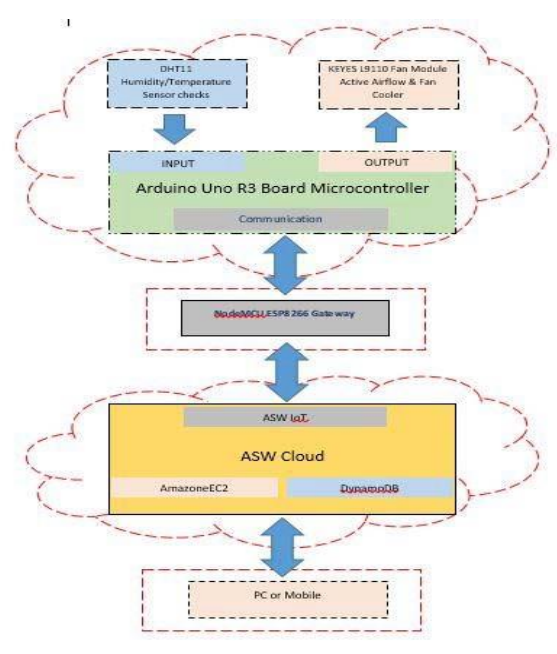

Figure 2. Auto Active Airflow-Fan Cooler model details

\subsection{System Architecture}

The main components used in this system are divided into three parts: Devices, Gateway, AWS Cloud computing, and Data transmission.

\subsubsection{Devices}

1) DHT11 Humidity/Temperature sensor, this module measures the surrounding air, and sends out a digital signal. It is calibrated with digital output of the temperature and humidity sensor. Accuracy for humidity is $20-80 \%$ and temperature is $0-50^{\circ} \mathrm{C}$. This sensor is used for measuring moisture content in the atmosphere. The sensor is shown in Figure 3.

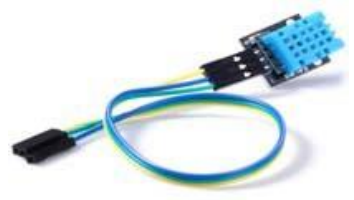

Figure 3. DHT11 Humidity and Temperature Sensor

2) KEYES L9110 Fan Module is for Arduino and experiments on DC motor, and uses IC L9110 as motor drive. This module is used for air controlling simulation, and is shown in Figure 4.

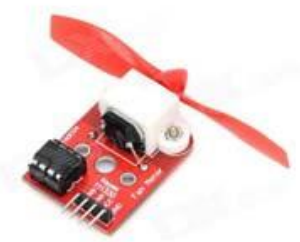

Figure 4. KEYES L9110 Fan Module

3) Arduino is a microcontroller board with open-source physical computing platform based on a simple I/O board that can connect to electrical circuits [3-4]. Arduino uses an ATmega328P chip as a microcontroller. We use it for Sensor Node to collect data, and it is networked by a NRF24L01 wireless communication system. The Ardunio board is shown in Figure 5.

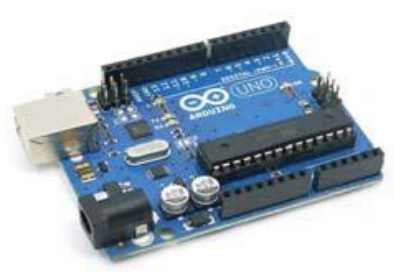

Figure 5. Arduino board

\subsubsection{Gateway}

An IoT gateway device is the communication between IoT devices, Sensors, Gateway and AWS cloud. By connecting the field device and AWS cloud, the IoT gateway devices operate local processing and storage solutions, has the ability to automatically control field devices based on data input by sensors. Node MCU is chosen for our purpose. It is a development board and part of the Internet of Things (IoT) [5]. The Node MCU board is based on ESP8266EX [6], which is a microcontroller integrated Wi-Fi receiver, and transmitter. The Node MCU board is shown in Figure 6.

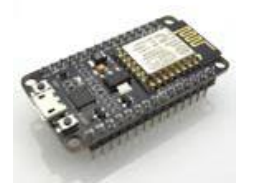

Figure 6. Node MCU

\subsubsection{AWS Cloud computing}

Cloud computing is a model that provides convenient, on-demand network access to a shared pool resources related functionality as a service (networks, servers, storage, applications) [7]. We choose Amazon Web Services (AWS) [8] which is a Public cloud type for the experiments. The AWS Cloud model is shown as Figure 7.

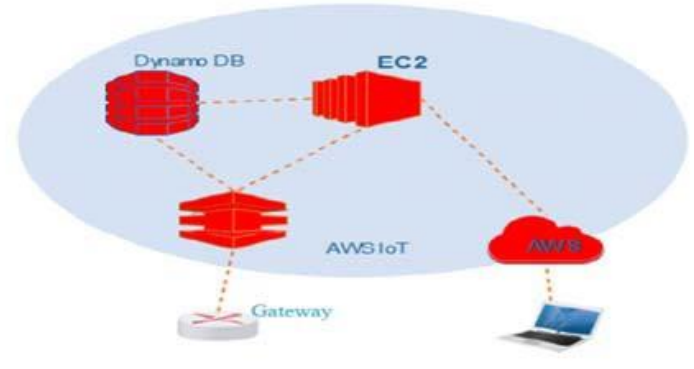

Figure 7. AWS Cloud Computing

The AWS Cloud model includes 1) Amazon Elastic Compute Cloud (EC2) for Application service and Web server; 2) AWS IoT is a IoT hub that connects with Gateway easily and securely to interact with Application service on EC2 [9]: with AWS IoT, sensor values can be transferred to store on DynamoDB; 3) DynamoDB [10] is a NoSQL database for collecting various sensor data (Temperature, Humidity and Fan status). These data will be stored in JSON format. In DynamoDB, we use NodeID as primary keys to identify uniquely each item in a table and Timestamps as a secondary index to provide grater querying flexibility.

\subsubsection{Data transmission}

Another feature of IoT is that the user can control and manage things from a wireless remote distance, and also on PC or mobile.

1) Monitoring: NodeMCU receives parameters detected by any Sensors from the Sensor node, and passes through the MQTT protocol to AWS IoT and transfers the data to DynamoDB. The monitoring data transmission is shown in Figure 8.

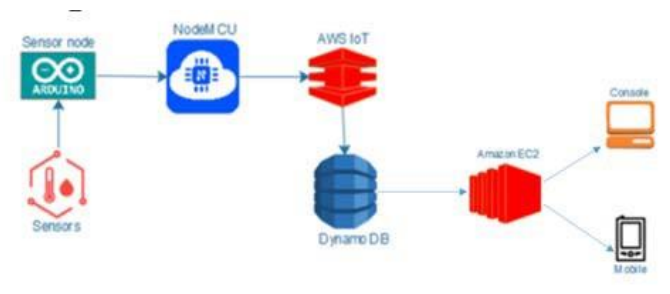

Figure 8. Monitoring Data Transmission

2) Controlling: Figure 11 shows the controlling data transmission. The user can control Fan via Web browser. If the user switches to the 
control function, EC2 (Application service + Web server) will send the request to AWS IoT, and pass into NodeMcU and Sensor node boards to enable the Fan. The controlling data transmission is shown in Figure 9.

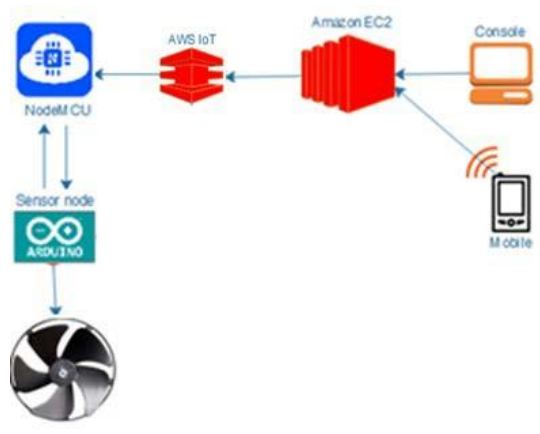

Figure 9. Controlling Data Transmission

\subsection{Protocols}

IoT requires a standard protocol. This system uses MQTT protocol [11][12]. MQTT is a machine-to- machine protocol. It is an extremely lightweight Publish/Subscribe messaging transport, as shown in Figure 10. It is useful for connections with remote locations, where a small code footprint is required with low network bandwidth (small size, low power usage, minimized data packets). For our model, it has been used in Gateway communicating to AWS IoT via the internet. In this work, Gateway is assigned to the Publisher, and AWS IoT to be the MQTT broker, while Application service is the Subscriber.

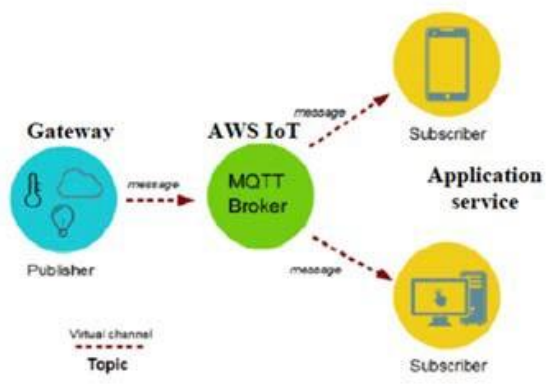

Figure 10. MQTT Protocol Model

\section{Results and Discussion}

Based on our experiments, we have simulated additional heated temperatures. By using a hair dryer, the temperature sensor is over 35 degrees. The motor must be driven at a speed of 50 , and greater than 45 degrees at 200 cycles. The motor can work well. When testing with NodeMCU, the NodeMCU cannot receive some values. However, this case occurs infrequently. Web browser displays the error message, as shown in Figure 11.
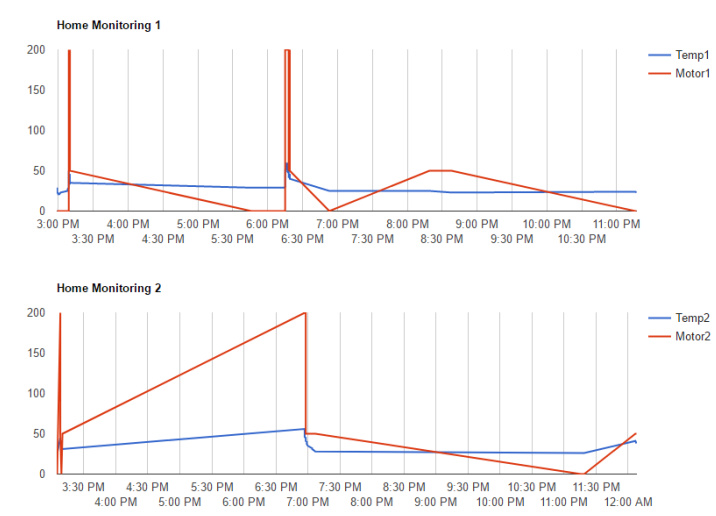

Figure 11. Motor onstantly

Sometimes, it is necessary to check NodeMCU for correcting values. When we press the reset bottom, these symptoms will disappear. For communications testing, the same communication channel is used without collision detection. NodeMCU cannot accept the values sometimes Home Monitoring 2 graph will notice that the Motor 2 value is constant from 3:30 PM to 7:00 PM, that is, the motor level increases from 50 to 200, which may not be accurate.

Refering to the information obtained from the results, we discussed in each subsystem where of was found that the overall systems, worked, as described in the manual. However, some improvements, such as position and number of sensors installed, may affect the accuracy of the measured data. Proper positioning and numbering should ensure that the measured data are accurate to meet the requirements.

\section{Conclusions}

This paper presented an IoT based designed of the Auto Active AirflowFan Cooler system. The model operates for data gathering and transmission using wireless internet under the Amazon Web Service, and preliminarys tests, showed that the developed model was capable of monitoring and controling devices in the environment, and had several advantages in terms of fast delivery, zero data lose, low cost, flexibility, and user friendliness. The user interfaces are simple and intuitive. The proposed project helps residents in real time monitoring of temperatures, and also reduces cost and expense sfor electricity.

For future work, this work can be applied, not just to ventilation, but also other projects, such as plant control, agricultural water control systems, and water monitoring systems, etc.

\section{References}

[1] J. Gubbi, R. Buyya, S. Marusic and M. Palaniswami, "Internet of Things (IoT): A vision, architectural elements, and future directions," Future Generation Computer Systems, Elsevier Science Publishers, September, 2013.

[2] D. Bandyopadhyay and J. Sen, "Internet of Things: Applications and Challenges in Technology and Standardization", J. Wireless Pers Commun, Kluwer Academic Publishers, Hingham, MA, USA, May 2011.

[3] Arduino, Arduino uno (2014), http://arduino.cc/en/Main/arduinoBoardUno.

[4] M. Margolis, Arduino Cookbook, O'Reilly Media, Inc., 2011.

[5] P. LechEmail and P. Włodarski, "Analysis of the IoT WiFi Mesh Network", In: Cybernetics and Mathematics Applications in Intelligent Systems (CSOC 2017), Advances in Intelligent Systems and Computing, vol 574, Springer, 2017.

[6] X. R. Jiang, Y. M. Lv, X. H. Cheng, "Design of Wireless Communication System Based on nRF24L01", Advanced Materials Research, Vols. 945949, pp. 1756-1759, 2014.

[7] P. Mell and T. Grance, "The NIST Definition of Cloud Computing", National Institute of Standards \&Technology, Gaithersburg, MD, United States, 2011

[8] Amazon Web Service (2017), https://aws.amazon.com

[9] Amazon Web Services (2017), "AWS IoT", https://aws.amazon.com/iot.

[10] J. Clark, "Amazon switches on DynamoDB cloud database service", ZDNet, 201 [11] "MQTT 3.1.1 specification", OASIS, December 10, 2015.

[12] A. Stanford-Clark and H. L. Truong, "MQTT For Sensor Networks (MQTT-SN) Protocol Specification Version 1.2", mqtt.org, p. 27, November 14, 2013. 